\title{
Wolbachia Detection in Field-Collected Mosquitoes from Cameroon
}

\author{
Roland Bamou 1,2@ , Adama Zan Diarra ${ }^{1,2}$, Marie Paul Audrey Mayi ${ }^{3}$, Borel Djiappi-Tchamen ${ }^{3,4}(\mathbb{D}$, \\ Christophe Antonio-Nkondjio ${ }^{4}(\mathbb{D})$ and Philippe Parola ${ }^{1,2, *}$
}

1 Aix Marseille Univ, IRD, AP-HM, SSA, VITROME, 13005 Marseille, France; bamou2011@gmail.com (R.B.); adamazandiarra@gmail.com (A.Z.D.)

2 IHU-Méditerranée Infection, 13005 Marseille, France

3 Vector Borne Diseases Laboratory of the Biology and Applied Ecology Research Unit (VBID-URBEA), Department of Animal Biology, Faculty of Science, University of Dschang, Dschang P.O. Box 067, Cameroon; mayimariepaulaudrey@yahoo.com (M.P.A.M.); borel_tchamen@yahoo.com (B.D.-T.)

4 Institut de Recherche de Yaoundé (IRY), Organisation de Coordination pour la Lutte Contre les Endémies en Afrique Centrale (OCEAC), Yaoundé P.O. Box 288, Cameroon; antonio_nk@yahoo.fr

* Correspondence: philippe.parola@univ-amu.fr

check for updates

Citation: Bamou, R.; Diarra, A.Z.; Mayi, M.P.A.; Djiappi-Tchamen, B.; Antonio-Nkondjio, C.; Parola, P. Wolbachia Detection in Field-Collected Mosquitoes from Cameroon. Insects 2021, 12, 1133. https://doi.org/ $10.3390 /$ insects 12121133

Academic Editor: Corey Brelsfoard

Received: 3 November 2021

Accepted: 14 December 2021

Published: 17 December 2021

Publisher's Note: MDPI stays neutral with regard to jurisdictional claims in published maps and institutional affiliations.

Copyright: (c) 2021 by the authors. Licensee MDPI, Basel, Switzerland. This article is an open access article distributed under the terms and conditions of the Creative Commons Attribution (CC BY) license (https:/ / creativecommons.org/licenses/by/ $4.0 /)$.
Simple Summary: Wolbachia bacteria from different strains, carried by many insects and nematodes, can interact in many ways with their hosts by changing their biology in different ways, including by suppressing vector population and reducing parasite transmission. Consequently, Wolbachia play an important role in vector control strategies. This study assessed the prevalence of natural Wolbachia infections in mosquitoes collected in Cameroon. Despite the low prevalence that was revealed, Wolbachia spp. were found in eight species of field-collected mosquitoes and are closely related to clades A and B. Aedes aegypti and A. gambiae sl., the main vectors of dengue and malaria, respectively, were not infected in this study, while C. moucheti recorded a high prevalence (46.67\%). Future characterisation of the Wolbachia bacteria obtained is needed.

Abstract: Wolbachia spp., known to be maternally inherited intracellular bacteria, are widespread among arthropods, including mosquitoes. Our study assessed the presence and prevalence of Wolbachia infection in wild mosquitoes collected in Cameroon, using the combination of 23s rRNA Anaplasmatacea and 16s rRNA Wolbachia genes. Mosquitoes that were positive for Wolbachia were sequenced for subsequent phylogenetic analysis. Out of a total of 1740 individual mosquitoes belonging to 22 species and five genera screened, 33 mosquitoes $(1.87 \%)$ belonging to eight species (namely, Aedes albopictus, A. contigus, Culex quinquefasciatus, C. perfuscus, C. wigglesworthi, C. duttoni, Anopheles paludis and Coquillettidia sp.) were found to be positive for Wolbachia infections. Wolbachia spp. were absent in A. gambiae and A. aegypti, the main vectors of malaria and dengue, respectively. Phylogenetic analysis of the $16 S$ RNA sequences showed they belong mainly to two distinct subgroups (A and B). This study reports the presence of Wolbachia in about eight species of mosquitoes in Cameroon and suggests that future characterisation of the strains is needed.

Keywords: Wolbachia infection; mosquito; phylogeny; 16s rRNA; 23s rRNA; Cameroon

\section{Introduction}

Mosquitoes (Diptera, Culicidae) are one of the most diverse groups of arthropods in the world and are found in a wide range of aquatic and terrestrial habitats with varying morphological and behavioural adaptations [1,2]. Their feeding behaviour as haematophagous insects gives them the ability to transmit a huge amount of pathogens, including viruses, bacteria, protozoa and nematodes from one vertebrate host to another [3]. In Cameroon, mosquitoes are implicated in the transmission of 26 arboviruses, malaria parasites and filarial worms causing diseases to humans, birds and great apes [4]. These diseases account for about $17 \%$ of infectious diseases in the world, and at least half of the world's 
population lives in areas where mosquito-borne diseases are endemic [4]. It has been noted that mosquitoes could be a vector of bacterial infectious diseases to humans, although only Rickettsia felis has been detected to date [5,6]. To reduce the burden of these threats, mosquito control measures have been developed, mainly based on chemical control using long-lasting insecticidal nets and indoor residual spraying [7]. Despite the measurable success of these tools in reducing the malarial burden, resistance to mosquito insecticides has emerged and now limits the effectiveness of these tools, calling for the development of new control strategies.

The genus Wolbachia includes maternally inherited endosymbiotic bacteria that naturally infect disparate ranges of insects, including mosquitoes of medical and veterinary importance [8-11]. It has long been thought that Wolbachia spp. were absent in some mosquitoes, such as A. aegypti and Anopheles gambiae mosquitoes [12]. However, recent studies have detected Wolbachia in many genera of mosquitoes including Aedes, Anopheles, Culex, Armigeres, Mansonia, Coquillettidia, Culiseta, Hodgesia, Ochlerotatus, Tripteroides and Uranotaenia [10,11,13-15]. Recently, the work of Ayala et al. [16] in Central Africa (Gabon) revealed that Wolbachia is largely prevalent among diverse groups of Anopheles species. Wolbachia may be distributed horizontally $[17,18]$ between mosquito populations and was also confirmed to be maternally transmitted within the A. moucheti population in Cameroon [10].

A recent study carried out in Italy highlighted the effect of this bacteria on the reduction in fertility in the arbovirus vector A. albopictus [19]. Experimental trials on the major malaria vector $A$. gambiae have also revealed the potential of Wolbachia to have an impact upon the replication of human malaria parasite Plasmodium falciparum $[6,17,18]$. Furthermore, Wolbachia have been reported to block the transmission of dengue, zika and yellow fever viruses [20-24].

Although these bacteria are highly prevalent (56\%) in A. moucheti malarial vectors in Cameroon, their distribution among other mosquito groups remains poorly understood [10]. In addition, knowledge about their prevalence remains limited. Data regarding Wolbachia in mosquitoes in Cameroon is lacking, and this study provides an opportunity to identify new Wolbachia strains that could be characterised. New Wolbachia strains are interesting candidates for vector control as they may confer useful phenotypes when transinfected into alternative mosquito hosts. In this study, the current prevalence of Wolbachia in different mosquito species was determined, and their sequences and phylogenetic were subsequently investigated using 23S rRNA Anaplasmataceae and Wolbachia 16S rRNA targeting $550 \mathrm{bp}$ and $460 \mathrm{bp}$ of the $16 S$ and $23 S$ genes, respectively.

We tested the hypothesis that different mosquito species from Cameroon may also display different levels of prevalence of Wolbachia endosymbiont infection.

\section{Materials and Methods}

\subsection{Biological Samples}

Field-collected mosquito samples were collected between 2017 and 2021 in different localities of Cameroon (Yaoundé, Dschang, Nyabessang) using different collection techniques, including the human landing catch (HLC), Centers for Disease Control light traps (CDC-LTs), sweep nets for adult collections and dipping for immature stages. CDC-LT collections were conducted both indoors and outdoors in four selected houses between $7 \mathrm{PM}$ and $6 \mathrm{AM}$ for at least three consecutive nights per season. Traps were placed indoors near someone sleeping under a net at about $1.5 \mathrm{~m}$ from the ground and outdoor on the veranda. The same number of houses were randomly selected per collection site for HLCs. In each house, indoor and outdoor mosquito collections were carried out between 7 PM and $6 \mathrm{AM}$ by two teams of two people per house using mouth aspirators. The two teams (indoor/outdoor collectors) exchanged positions in each homestead every hour of the night. Mosquito collection bags were exchanged hourly. Immature mosquito stages at breeding sites (abandoned tyres, metal and plastic containers, gutters and stagnant water pools) were collected using the dipping technique, while sweep nets were used to catch 
adult mosquitoes resting on vegetation. The mosquitoes collected belonged to the genera Anopheles, Culex, Aedes, Lutzia, Mansonia, Coquillettidia and Eretmapodites. Each mosquito species was first identified morphologically using a binocular microscope and identification keys [25-27], and this was later confirmed by matrix-assisted laser desorption ionizationtime of flight mass spectrometry (MALDI-TOF MS) and molecular analysis [28]. Damaged and unidentified specimens (due to the absence of diagnostic characteristics and the loss of legs for MALDI-TOF MS identification) were identified using molecular tools. Mosquitoes were stored either in silicate or in empty containers (Eppendorf tubes or petri dishes with no preserving product) at room temperature, or in silicate, RNAlater, $70 \%$ alcohol and in empty containers in the fridge at $-20{ }^{\circ} \mathrm{C}$ for two to 48 months, depending on the time of collection.

Using a sterile surgical blade, mosquito legs were removed and analysed using MALDI-TOF MS for species identification. The rest of the mosquito body, and at times the whole mosquito, were used for molecular identification and microbial detection.

\subsection{MALDI TOF MS Analyses and Molecular Identification of Mosquitoes}

The identification of the mosquito species was confirmed by MALDI-TOF MS and molecular biology analyses, and these results have been presented elsewhere. Briefly, the legs of specimens were introduced to $1.5 \mathrm{~mL}$ Eppendorf tubes containing $20 \mu \mathrm{L}$ of protein extraction mix (70\% formic acid $(v / v)$ and $50 \%$ acetonitrile $(v / v))$ and grinded using glass beads in a Tissue Lyser [29,30]. One microlitre of each protein extract was deposited on a target plate in quadruplicate, and one microlitre of matrix was added to each spot and allowed to dry. Protein mass spectra were obtained using a Microflex LT MALDI-TOF mass spectrometer (Bruker Daltonics, Bremen, Germany) [31] and crosswise tested against an in-house database available at VITROME, containing spectra of known mosquito species. The results are presented by the reliability of species identification, estimated as the log score value (LSV) and calculated using a biostatistical algorithm from the MALDI Biotyper software v.3.0. The LSV range from 0-3 and values equal to or greater than 1.8 are considered as threshold values for the species identification of mosquitoes. Species that were not identified through morphological and MALDI-TOF MS methods or with no agreement between the methods were further analysed by molecular biology using cytochrome oxidase subunit 1 for invertebrate Cox 1 genes [28]. DNA was individually extracted from the carcasses (specimens without legs) of mosquito specimens $(n=1740)$ using the QIAamp DNA tissue extraction kit (Qiagen, Hilden, Germany), according to the manufacturer's instructions.

\subsection{Mosquito Microbiota Analysis}

Mosquito samples were submitted to five bacteria screening groups, namely Rickettsia spp., Borrelia spp., Bartonella spp., Coxiella burnetii and Anaplasmataceae. Mosquitoes were pooled (4-10 individual mosquitoes) and screened for the detection of Rickettsia spp., Borrelia spp., Bartonella spp., Coxiella burnetii and Anaplasmataceae using quantitative PCR (qPCR) [32,33]. When a pool was found to be positive for one type of bacteria, specimens were then analysed individually using the same method. Target genes, primers and probe sequences (Table 1) were used as previously described in other studies [32,33]. DNA from laboratory-cultured strains of Borrelia crocidurae, Rickettsia africae, Bartonella quintana, Coxiella burnetii and Ehrlichia canis were used as positive controls. DNA free of pathogens from laboratory-reared ticks was used as a negative control. Samples were considered to be positive when the $\mathrm{Ct}<36$ for all bacteria tested. Later on, samples (from individual specimens) positive for Anaplasmataceae underwent standard PCR amplification and sequencing of the 23s rRNA and 16s rRNA genes, as described by Diarra et al. [32]. 
Table 1. Primers and probes used for real-time quantitative and standard PCR in this study.

\begin{tabular}{|c|c|c|c|}
\hline Microorganisms & \multicolumn{2}{|c|}{ Targeted Sequence } & Primers F, R $\left(5^{\prime}-3^{\prime}\right)$ and Probes p (6FAM-TAMRA) \\
\hline \multicolumn{4}{|c|}{ qPCR primers } \\
\hline \multirow{3}{*}{ Anaplasmataceae } & \multirow{3}{*}{$23 S$} & TtAna_F & TGACAGCGTACCTTTTGCAT \\
\hline & & TtAna_R & GTAACAGGTTCGGTCCTCCA \\
\hline & & TtAna_P & 6FAM-GGATTAGACCCGAAACCAAG \\
\hline \multirow{3}{*}{ Bartonella } & \multirow{3}{*}{ ITS } & Barto_ITS2_F & GGGGCCGTAGCTCAGCTG \\
\hline & & Barto_ITS2_R & TGAATATATCTTCTCTTCACAATTTC \\
\hline & & Barto_ITS2_P & 6FAM-CGATCCCGTCCGGCTCCACCA \\
\hline \multirow{6}{*}{ Borrelia } & \multirow{3}{*}{$16 S$} & Bor_16S_3F & AGCCTTTAAAGCTTCGCTTGTAG \\
\hline & & Bor_16S_3R & GCCTCCCGTAGGAGTCTGG \\
\hline & & Bor_16S_3P & 6FAM-CCGGCCTGAGAGGGTGAACGG \\
\hline & \multirow{3}{*}{ ITS4 } & Bor_ITS4_F & GGCTTCGGGTCTACCACATCTA \\
\hline & & Bor_ITS4_R & CCGGGAGGGGAGTGAAATAG \\
\hline & & Bor_ITS4_P & 6FAM-TGCAAAAGGCACGCCATCACC \\
\hline \multirow{6}{*}{ Coxiella burnetii } & \multirow{3}{*}{ IS1111A } & CB_IS1111_0706F & CAAGAAACGTATCGCTGTGGC \\
\hline & & CB_IS1111_0706R & CACAGAGCCACCGTATGAATC \\
\hline & & CB_IS1111_0706P & 6FAM-CCGAGTTCGAAACAATGAGGGCTG \\
\hline & \multirow{3}{*}{$\begin{array}{l}\text { Hyp. Protein } \\
\text { IS30A }\end{array}$} & CB_IS30A_3F & CGCTGACCTACAGAAATATGTCC \\
\hline & & CB_IS30A_3R & GGGGTAAGTAAATAATACCTTCTGG \\
\hline & & CB_IS30A_3P & 6FAM-CATGAAGCGATTTATCAATACGTGTATGC \\
\hline \multirow{3}{*}{ Rickettsia spp. } & \multirow{3}{*}{ glt $A$} & RKND03_F & GTGAATGAAAGATTACACTATTTAT \\
\hline & & RKND03_R & GTATCTTAGCAATCATTCTAATAGC \\
\hline & & RKND03 P & 6FAM-CTATTATGCTTGCGGCTGTCGGTTC \\
\hline \multicolumn{4}{|c|}{ Standard PCR primers } \\
\hline \multirow{2}{*}{$\begin{array}{l}\text { Invertebrate identification } \\
\text { (Folmer) }\end{array}$} & \multirow{2}{*}{ COI } & LCO1490 & GGTCAACAAATCATAAAGATATTGG \\
\hline & & HCO2198 & TAAACTTCAGGGTGACCAAAAAATCA \\
\hline \multirow{2}{*}{ Anaplasmataceae } & \multirow{2}{*}{$23 S$} & Ana23S-212f & ATAAGCTGCGGGGAATTGTC \\
\hline & & Ana23S-753r & TGCAAAAGGTACGCTGTCAC \\
\hline \multirow{2}{*}{ Wolbachia } & \multirow{2}{*}{$16 S$} & W-SpecF & CATACCTATTCGAAGGGATAG \\
\hline & & W-SpecR & AGCTTCGAGTGAA ACCAATTC \\
\hline
\end{tabular}

\subsection{Sequencing and Phylogenetic Analyses}

Sequencing and phylogenetic analyses of positive samples (33) was performed as described elsewhere [34] using the 16S rRNA and 23S rRNA genes targeting 550bp and $460 \mathrm{bp}$ of the $16 S$ and $23 S$ genes, respectively. The products obtained were visualised on $1.5 \%$ agarose gel stained with SYBR Safe and purified using a Macherey Nagel (NucleoFast 96 PCR, Düren, Germany) plate. Sequencing was performed using the BigDye Terminator v1.1, v3.1 5x Sequencing Buffer (Applied Biosystems, Warrington, UK) and run on an automated sequencer. The sequence chromatograms that were obtained were assembled and edited using Chromas Pro1.77 (Technelysium Pty Ltd., Tewantin, Australia). The sequences obtained were used to perform BLAST searches against the National Center for Biotechnology Information (NCBI) GenBank database and were then aligned using BioEdit. A phylogenetic tree was constructed and edited using the maximum likelihood method with the model selection determined by TOPALI v2.5 and MEGA11, respectively. 
Statistical support for internal branches of the trees was evaluated by bootstrapping with 1000 iterations.

\section{Results}

In total, about 1740 mosquitoes collected in the field were analysed, and their abundance varied with the species. Twenty-two species belonging to five genera were identified and confirmed by a molecular analysis tool or MALDI TOF MS. In this paper, we focus on the detection of mosquito-borne bacteria circulating in Cameroon.

From the five bacterial groups screened in this study (Rickettsia spp., Borrelia spp., Bartonella spp., Coxiella burnetii and Anaplasmataceae), only DNA particles of Anaplasmataceae were found in our samples $(33 / 1740 ; 1.89 \%)$. Infected samples included Anopheles spp. (A. paludis), Aedes spp. (A. albopictus, A. contigus), Culex spp. (C. quinquefasciatus, C. moucheti, C. wigglesworthi, C. perfuscus), Lutzia spp. (L. tigripes) and Coquillettidia spp. The prevalence of infection according to mosquito species is presented in Table 2. Following sequencing, all positive samples (33) using the 23s $r R N A$ gene were found to be positive for Wolbachia spp. (W. pipientis, Wolbachia endosymbiont of Ctenocephalides felis wCfeT, Wolbachia endosymbiont of Chrysomya megacephala) with the exception of two samples that were positive for Anaplasma ovis (MT408585) with identification scores ranging from 99.40-100\%. The top hit accession numbers of positive samples in this case were CP037426, CP021120, CP051156, CP050530 and KT827385. In contrast, after sequencing with the 16s rRNA gene, the positive samples were made up of Wolbachia spp. (32) and one uncultured bacterium. For the 16s rRNA gene, the top hit accession numbers were KX155505, MK184237, MN123078, CP041923, MH447384, AB508951.1 and KT273278. Both positive samples found with Anaplasma ovis (MT408585) using the 23s rRNA gene were identified as Wolbachia spp. (AB508951 and KX155505) using the 16s rRNA gene (Table S1). Similarity varies from $97.5-100 \%$ and $98.2-100 \%$ after BLAST query on GenBank using the 16s $r R N A$ and $23 s$ rRNA genes, respectively.

Table 2. Prevalence of Wolbachia spp. detected in mosquitoes collected in Cameroon.

\begin{tabular}{ccc}
\hline Species & Tested & Wolbachia Positive (\%) \\
\hline Anopheles gambiae s.l. & 171 & $0(0)$ \\
Anopheles paludis & 211 & $1(0.4 \%)$ \\
Anopheles nili & 2 & $0(0)$ \\
Anopheles moucheti & 20 & $0(0)$ \\
Culex (Lutzia) tigripes & 18 & $1(5.55)$ \\
Culex duttoni & 43 & $0(0)$ \\
Culex perfuscus & 116 & $4(3.44)$ \\
Culex quinquefasciatus & 730 & $7(0.95)$ \\
Culex univittatus & 11 & $0(0)$ \\
Culex moucheti & 15 & $7(46.67)$ \\
Culex wigglesworthi & 1 & $1(100)$ \\
Culex sp. & 52 & $2(3.84)$ \\
Aedes albopictus & 155 & $8(5.16)$ \\
Aedes aegypti & 43 & $0(0)$ \\
Aedes africanus & 4 & $0(0)$ \\
Aedes simpsoni & 6 & $0(0)$ \\
Aedes contigus & 32 & $1(3.12)$ \\
Aedes sp. & 6 & $0(0)$ \\
Coquillettidia spp. & 7 & $1(14.28)$ \\
Mansonia africana & 42 & $0(0)$ \\
Mansonia uniformis & 55 & $0(0)$ \\
Total & 1740 & $33(1.89)$ \\
\hline
\end{tabular}

Figure 1 shows the resulting $16 \mathrm{~s} r R N A$ gene tree. This tree indicates that the majority of Wolbachia identified in this study are more closely related to each other than to other known Wolbachia strains included as references (Figure 1). Out of 17 sequences included 
in the phylogenetic analysis, six (35.29\%) clustered with Clade B while three (17.64\%) clustered with Clade A, and one clustered (5.88\%) with Clade T. The remainder (seven sequences, $41.17 \%$ ) were not well grouped according to the clusters.

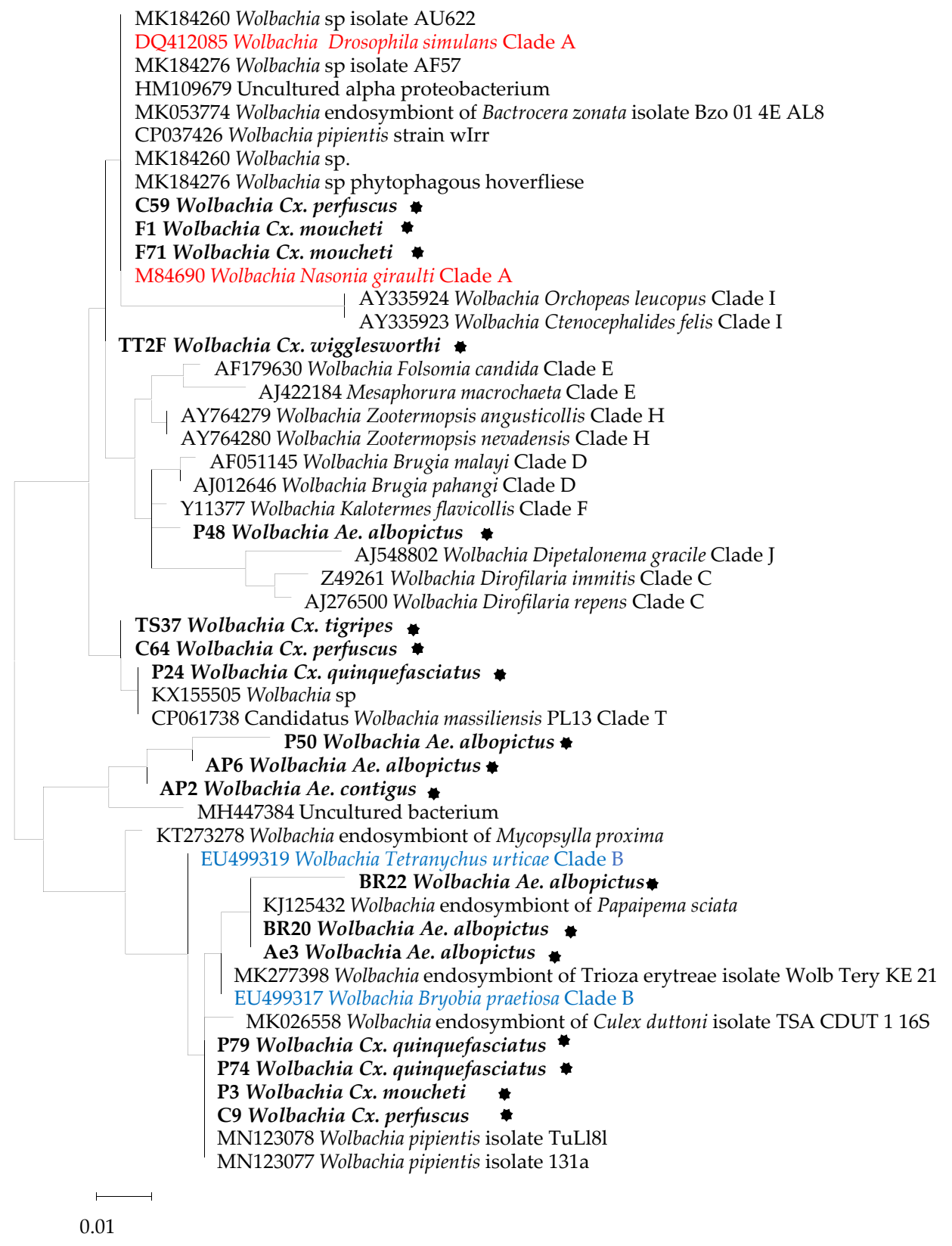

Figure 1. Wolbachia strain phylogenetic analysis using the $16 S$ rRNA gene. The evolutionary history was inferred using the maximum likelihood method and Tamura-Nei model [35]. The tree with the highest log likelihood $(-1118,14)$ is shown. The initial tree(s) for the heuristic search were obtained automatically by applying the neighbour-joining and BioNJ algorithms to a matrix of pairwise distances estimated using the Tamura-Nei model, and then by selecting the topology with the higher log likelihood value. The tree is drawn to scale, with branch lengths measured in the number of substitutions per site. This analysis involved 61 nucleotide sequences. There were a total of 350 positions in the final [13] dataset. Evolutionary analyses were conducted in MEGA11 [36]. Sequences from this study are indicated in bold, while the two main groups, Clade A (red) and Clade B (blue), are coloured. The Wolbachia strains obtained in this study are in bold and marked with a black star. 


\section{Discussion}

In this study, we demonstrated the natural infection of this endosymbiont in fieldcollected mosquitoes in Cameroon through the combination of qPCR, standard PCR-based Wolbachia screening and sequencing (using the $16 s$ r $R N A$ and $23 s$ r $r N A$ genes). To the best of our knowledge, Wolbachia has thus far only been detected in A. mouchet in Cameroon [10]. The successful detection of this bacteria through the 16s $r R N A$ gene compared to standard detection of the specific gene of Wolbachia $(w s p)$ has increased the evidence of the presence of this bacteria in Anopheles mosquitoes [8-10,37,38].

In general, the prevalence of infection was low compared to that which has been reported in other studies in Africa, with $56.6 \%$ of A. moucheti in Cameroon [10]. Previous studies revealed a prevalence ranging from $3.14-7.75 \%$ in Tanzania [37], 28.1\% in Singapore [39], 37.1\% in Thailand [40] and 26.38\% in Sri Lanka [13] for multiple mosquito species. This low infection rate may be due either to environmental differences between areas or to the potential low density of Wolbachia in our mosquitoes, as observed elsewhere [41,42]. It is also common to see varying Wolbachia infection rates from the same insect host collected at different geographical locations, as in the case of A. aegypti from New Mexico (57.4\%), Texas $(0 \%)$ and Florida (4.75\%) [43], or the case of A. demeilloni, where Wolbachia was detected in $38.7 \%$ of specimens from Kenya, and $89.3 \%$ and $100 \%$ in specimens from DRC in 2015 and 2019, respectively [10].

None of the major malaria vectors were found to harbour Wolbachia, except the secondary vector $A$. paludis $(1 / 177)$ collected in Nyabessan in 2016. To our knowledge, this is the first time that Wolbachia spp. has been detected in this species in Africa or elsewhere. This low detection of Wolbachia in Anopheles mosquitoes aligns with other studies [41], strengthening the evidence of their incompatibility with anophelines and revealing possible contamination from environmental sources such as breeding water.

More Culex mosquitoes were found to be infected by Wolbachia, but the prevalence was low compared to other studies $[11,13]$. The species screened for bacterial infection in this study included C. moucheti (vector of Ntaya virus), C. quinquefasciatus (potential vector of bancrofti filariasis and arboviruses such as West Nile, Babanki and Western equine encephalitis viruses), C. duttoni (vector of Arb11266), C. perfuscus (possible vector of Zika virus), C. univittatus (vector of West Nile, Usutu, Wesselsbron, Sindbis, Rift valley fever and Spondweni viruses) and L. tigripes (vector of Ntaya, Kamese, Mossuril, Sindbis and Babanki viruses). Wolbachia-infected species included C. quinquefasciatus, C. moucheti, C. perfuscus and C. wigglesworthi. Previous studies reported Wolbachia in C. quinquefasciatus [11,13,44]. Regarding the other species, this is the first time they have been detected with these bacteria. The bacterial infection of mosquitoes may vary in space and time. In Thailand, Culex mosquitoes were not infected by Wolbachia [45], while the study by Wiwatanaratnabutr [40] revealed Wolbachia infections in C. tritaeniorhynchus and C. gelidus.

Although previous studies reported the natural infection of Wolbachia in both major vectors of dengue and zika, A. aegypti and A. albopictus [13,43,46], this study reported a low prevalence of infection only in the invasive mosquito A. albopictus. In addition, one sample of $A$. contigus was found to be infected. Wolbachia-infected Coquillettidia mosquitoes were also observed for the first time.

\section{Conclusions}

This study revealed the natural presence or evidence of Wolbachia in field-collected mosquitoes from Cameroon. Albeit at a very low frequency (1.87\%), Wolbachia was detected in A. albopictus, A. contigus, C. moucheti, C. quinquefasciatus, C. perfuscus, C. wigglesworthi and Coquillettidia spp. Future studies are needed to characterise these strains and to determine the impact they might have on disease transmission. The exploration of other areas in order to have an understanding of diverse and consistent mosquito populations should also take place, with the aim of building a greater understanding of species carrying Wolbachia, since very few specimens and samples were screened for some mosquito species from this study, which is not representative of the population of the species in question. 
Supplementary Materials: The following are available online at https:/ / www.mdpi.com/article / 10.3390 /insects12121133/s1, Table S1: Blast results of selected specimens showing cover, percent identification, accession number and species of closely similar bacteria using both 16s $r R N A$ and $23 \mathrm{~s}$ rRNA genes.

Author Contributions: Conceived and designed the experiments: R.B., P.P. Performed the experiments: R.B., A.Z.D. Analysed the data: R.B., A.Z.D. Contributed reagents/materials: M.P.A.M., B.D.-T., A.Z.D., C.A.-N., P.P. Drafted the paper: R.B. Critically revised the paper: all the authors. All authors have read and agreed to the published version of the manuscript.

Funding: This work received financial support from the Institut Hospitalo-Universitaire (IHU) Méditerranée Infection, the National Research Agency under the "Investissements d'avenir" programme, reference ANR-10-IAHU-03, the Région Provence Alpes Côte d'Azur and European FEDER PRIMI funding. The funding body did not have any role in the design, data collection, analysis and interpretation or in the writing up of the manuscript.

Institutional Review Board Statement: The study was conducted under ethical clearance No. 2018/06/1036/CE/CNERSH/SP and No. 1284/CRERSHC/2021, granted by the Cameroon National (CNE) and Centre Regional (CRE) Ethics Committee for Research on Human Health.

Informed Consent Statement: Authorisation to carry out the study was obtained from the authorities and from the heads of households through an informed consent form. The volunteer collectors were adults living in the collection sites. After each collection performed using human landing catch, malaria prophylaxis was given to volunteer collectors. Mosquitoes were transported to VITROME according to importation authorisation No. ER-22-2020.

Data Availability Statement: All the data from the study is available in the manuscript.

Acknowledgments: We are grateful to the researchers from OCEAC and VBID-URBEA who were involved in mosquito collection and identification.

Conflicts of Interest: The authors declare that they have no competing interest.

\section{References}

1. Sinka, M.E.; Bangs, M.J.; Manguin, S.; Rubio-Palis, Y.; Chareonviriyaphap, T.; Coetzee, M.; Mbogo, C.M.; Hemingway, J.; Patil, A.P.; Temperley, W.H.; et al. A Global Map of Dominant Malaria Vectors. Parasites Vectors 2012, 5, 69. [CrossRef]

2. Becker, N.; Petrić, D.; Zgomba, M.; Boase, C.; Madon, M.; Dahl, C.; Kaiser, A. Medical Importance of Mosquitoes. In Mosquitoes and Their Control; Springer: Berlin/Heidelberg, Germany, 2010; pp. 25-42. [CrossRef]

3. Azari-Hamidian, S.; Norouzi, B.; Harbach, R.E. A Detailed Review of the Mosquitoes (Diptera: Culicidae) of Iran and Their Medical and Veterinary Importance. Acta Trop. 2019, 194, 106-122. [CrossRef]

4. Bamou, R.; Mayi, M.P.A.; Djiappi-Tchamen, B.; Nana-Ndjangwo, S.M.; Nchoutpouen, E.; Cornel, A.J.; Awono-Ambene, P.; Parola, P.; Tchuinkam, T.; Antonio-Nkondjio, C. An Update on the Mosquito Fauna and Mosquito-Borne Diseases Distribution in Cameroon. Parasites Vectors 2021, 14, 1-15. [CrossRef]

5. $\quad$ Barua, S.; Hoque, M.M.; Kelly, P.J.; Poudel, A.; Adekanmbi, F.; Kalalah, A.; Yang, Y.; Wang, C. First Report of Rickettsia felis in Mosquitoes, USA. Emerg. Microbes Infect. 2020, 9, 1008-1010. [CrossRef]

6. Dieme, C.; Bechah, Y.; Socolovschi, C.; Audoly, G.; Berenger, J.-M.; Faye, O.; Raoult, D.; Parola, P. Transmission Potential of Rickettsia felis Infection by Anopheles gambiae Mosquitoes. Proc. Natl. Acad. Sci. USA 2015, 112, 201413835. [CrossRef] [PubMed]

7. WHO. World Malaria Report; World Health Organization: Geneva, Switzerland, 2020. Available online: https://www.who.int/ publications/i/item/9789240015791 (accessed on 22 December 2020).

8. Gomes, F.M.; Hixson, B.L.; Tyner, M.D.W.; Ramirez, J.L.; Canepa, G.E.; Alves e Silva, T.L.; Molina-Cruz, A.; Keita, M.; Kane, F.; Traoré, B.; et al. Effect of Naturally Occurring Wolbachia in Anopheles gambiae s.l. Mosquitoes from Mali on Plasmodium falciparum Malaria Transmission. Proc. Natl. Acad. Sci. USA 2017, 114, 12566. [CrossRef] [PubMed]

9. Jeffries, C.L.; Lawrence, G.G.; Golovko, G.; Kristan, M.; Orsborne, J.; Spence, K.; Hurn, E.; Bandibabone, J.; Tantely, L.M.; Raharimalala, F.N.; et al. Novel Wolbachia Strains in Anopheles Malaria Vectors from Sub-Saharan Africa. Wellcome Open Res. 2018, 3, 113. [CrossRef] [PubMed]

10. Walker, T.; Quek, S.; Jeffries, C.L.; Bandibabone, J.; Dhokiya, V.; Bamou, R.; Kristan, M.; Messenger, L.A.; Gidley, A.; Hornett, E.A.; et al. Stable High-Density and Maternally Inherited Wolbachia Infections in Anopheles moucheti and Anopheles demeilloni Mosquitoes. Curr. Biol. 2021, 31, 2310-2320.e5. [CrossRef]

11. Wong, M.L.; Liew, J.W.K.; Wong, W.K.; Pramasivan, S.; Mohamed Hassan, N.; Wan Sulaiman, W.Y.; Jeyaprakasam, N.K.; Leong, C.S.; Low, V.L.; Vythilingam, I. Natural Wolbachia Infection in Field-Collected Anopheles and Other Mosquito Species from Malaysia. Parasites Vectors 2020, 13, 414. [CrossRef] [PubMed] 
12. Kittayapong, P.; Milne, J.R.; Tigvattananont, S.; Baimai, V. Distribution of the Reproduction-Modifying Bacteria, Wolbachia, in Natural Populations of Tephritid Fruit Flies in Thailand. Sci. Asia 2000, 26, 93-103. [CrossRef]

13. Nugapola, N.W.N.P.; De Silva, W.A.P.P.; Karunaratne, S.H.P.P. Distribution and Phylogeny of Wolbachia Strains in Wild Mosquito Populations in Sri Lanka. Parasites Vectors 2017, 10, 230. [CrossRef] [PubMed]

14. Werren, J.H.; Baldo, L.; Clark, M.E. Wolbachia: Master Manipulators of Invertebrate Biology. Nat. Rev. Microbiol. $2008,6,741-751$. [CrossRef] [PubMed]

15. Zug, R.; Hammerstein, P. Still a Host of Hosts for Wolbachia: Analysis of Recent Data Suggests That $40 \%$ of Terrestrial Arthropod Species Are Infected. PLoS ONE 2012, 7, e38544. [CrossRef]

16. Ayala, D.; Akone-Ella, O.; Rahola, N.; Kengne, P.; Ngangue, M.F.; Mezeme, F.; Makanga, B.K.; Nigg, M.; Costantini, C.; Simard, F.; et al. Natural Wolbachia Infections Are Common in the Major Malaria Vectors in Central Africa. Evol. Appl. 2019, 12, 1583-1594. [CrossRef] [PubMed]

17. Chrostek, E.; Pelz-Stelinski, K.; Hurst, G.D.D.; Hughes, G.L. Horizontal Transmission of Intracellular Insect Symbionts via Plants. Front. Microbiol. 2017, 8, 2237. [CrossRef] [PubMed]

18. Ahmed, M.Z.; Breinholt, J.W.; Kawahara, A.Y. Evidence for Common Horizontal Transmission of Wolbachia among Butterflies and Moths. BMC Evol. Biol. 2016, 16, 118. [CrossRef] [PubMed]

19. Caputo, B.; Moretti, R.; Manica, M.; Serini, P.; Lampazzi, E.; Bonanni, M.; Fabbri, G.; Pichler, V.; Torre, A.; Calvitti, M. A Bacterium against the Tiger: Preliminary Evidence of Fertility Reduction after Release of Aedes albopictus Males with Manipulated Wolbachia Infection in an Italian Urban Area. Pest Manag. Sci. 2020, 76, 1324-1332. [CrossRef] [PubMed]

20. Dutra, H.L.C.; Rocha, M.N.; Dias, F.B.S.; Mansur, S.B.; Caragata, E.P.; Moreira, L.A. Wolbachia Blocks Currently Circulating Zika Virus Isolates in Brazilian Aedes aegypti Mosquitoes. Cell Host Microbe 2016, 19, 771-774. [CrossRef]

21. Caragata, E.P.; Dutra, H.L.C.; Moreira, L.A. Inhibition of Zika Virus by Wolbachia in Aedes aegypti. Microb. Cell 2016, 3, $293-295$. [CrossRef]

22. Pinto, S.B.; Riback, T.I.S.; Sylvestre, G.; Costa, G.; Peixoto, J.; Dias, F.B.S.; Tanamas, S.K.; Simmons, C.P.; Dufault, S.M.; Ryan, P.A.; et al. Effectiveness of Wolbachia-Infected Mosquito Deployments in Reducing the Incidence of Dengue and Other Aedes-Borne Diseases in Niterói, Brazil: A Quasi-Experimental Study. PLoS Negl. Trop. Dis. 2021, 15, e0009556. [CrossRef]

23. Frentiu, F.D.; Zakir, T.; Walker, T.; Popovici, J.; Pyke, A.T.; van den Hurk, A.; McGraw, E.A.; O’Neill, S.L. Limited Dengue Virus Replication in Field-Collected Aedes aegypti Mosquitoes Infected with Wolbachia. PLoS Negl. Trop. Dis. 2014, 8, e2688. [CrossRef]

24. Walker, T.; Johnson, P.H.; Moreira, L.A.; Iturbe-Ormaetxe, I.; Frentiu, F.D.; McMeniman, C.J.; Leong, Y.S.; Dong, Y.; Axford, J.; Kriesner, P.; et al. The WMel Wolbachia Strain Blocks Dengue and Invades Caged Aedes aegypti Populations. Nature 2011, 476, 450-453. [CrossRef] [PubMed]

25. Coetzee, M. Key to the Females of Afrotropical Anopheles Mosquitoes (Diptera: Culicidae). Malar. J. 2020, 19, 70. [CrossRef] [PubMed]

26. Gillies, M.T. A Supplement to the Anophelinae of Africa South of the Sahara (Afrotropical Region). Publ. S. Afr. Inst. Med. Res. 1987, 55, 1-143.

27. Jupp, P. Mosquitoes of Southern Africa; Ekogilde Publishers: Johannesburg, South Africa, 1996; p. 156.

28. Folmer, O.; Black, M.; Hoeh, W.; Lutz, R.; Vrijenhoek, R. DNA Primers for Amplification of Mitochondrial Cytochrome c Oxidase Subunit I from Diverse Metazoan Invertebrates. Mol. Mar. Biol. Biotechnol. 1994, 3, 294-299.

29. Fall, F.K.; Laroche, M.; Bossin, H.; Musso, D.; Parola, P. Performance of MALDI-TOF Mass Spectrometry to Determine the Sex of Mosquitoes and Identify Specific Colonies from French Polynesia. Am. J. Trop. Med. Hyg. 2021, 104, tpmd200031. [CrossRef] [PubMed]

30. Tandina, F.; Niaré, S.; Laroche, M.; Koné, A.K.; Diarra, A.Z.; Ongoiba, A.; Berenger, J.M.; Doumbo, O.K.; Raoult, D.; Parola, P. Using MALDI-TOF MS to Identify Mosquitoes Collected in Mali and Their Blood Meals. Parasitology 2018, 145, 1170-1182. [CrossRef] [PubMed]

31. Yssouf, A.; Almeras, L.; Raoult, D.; Parola, P. Emerging Tools for Identification of Arthropod Vectors. Future Microbiol. 2016, 11, 549-566. [CrossRef] [PubMed]

32. Diarra, A.Z.; Almeras, L.; Laroche, M.; Berenger, J.-M.; Kone, A.K.; Bocoum, Z.; Dabo, A.; Doumbo, O.; Raoult, D.; Parola, P. Molecular and MALDI-TOF Identification of Ticks and Tick-Associated Bacteria in Mali. PLoS Negl. Trop. Dis. 2017, 11, e0005762. [CrossRef] [PubMed]

33. Sadeddine, R.; Diarra, A.Z.; Laroche, M.; Mediannikov, O.; Righi, S.; Benakhla, A.; Dahmana, H.; Raoult, D.; Parola, P. Molecular Identification of Protozoal and Bacterial Organisms in Domestic Animals and Their Infesting Ticks from North-Eastern Algeria. Ticks Tick-Borne Dis. 2020, 11, 101330. [CrossRef] [PubMed]

34. Hamlili, F.Z.; Thiam, F.; Laroche, M.; Diarra, A.Z.; Doucouré, S.; Gaye, P.M.; Fall, C.B.; Faye, B.; Sokhna, C.; Sow, D.; et al. MALDI-TOF Mass Spectrometry for the Identification of Freshwater Snails from Senegal, Including Intermediate Hosts of Schistosomes. PLoS Negl. Trop. Dis. 2021, 15, e0009725. [CrossRef] [PubMed]

35. Tamura, K.; Nei, M. Estimation of the Number of Nucleotide Substitutions in the Control Region of Mitochondrial DNA in Humans and Chimpanzees. Mol. Biol. Evol. 1993, 10, 512-526. [CrossRef] [PubMed]

36. Tamura, K.; Stecher, G.; Kumar, S. MEGA11: Molecular Evolutionary Genetics Analysis Version 11. Mol. Biol. Evol. 2021, 38, 3022-3027. [CrossRef] 
37. Baldini, F.; Rougé, J.; Kreppel, K.; Mkandawile, G.; Mapua, S.A.; Sikulu-Lord, M.; Ferguson, H.M.; Govella, N.; Okumu, F.O. First Report of Natural Wolbachia Infection in the Malaria Mosquito Anopheles arabiensis in Tanzania. Parasites Vectors 2018, 11, 1-7. [CrossRef] [PubMed]

38. Niang, E.H.A.; Bassene, H.; Makoundou, P.; Fenollar, F.; Weill, M.; Mediannikov, O. First Report of Natural Wolbachia Infection in Wild Anopheles funestus Population in Senegal. Malar. J. 2018, 17, 408. [CrossRef]

39. Kittayapong, P.; Baisley, K.J.; Baimai, V.; O'Neill, S.L. Distribution and Diversity of Wolbachia Infections in Southeast Asian Mosquitoes (Diptera: Culicidae). J. Med. Entomol. 2000, 37, 340-345. [CrossRef]

40. Wiwatanaratanabutr, I. Geographic Distribution of Wolbachial Infections in Mosquitoes from Thailand. J. Invertebr. Pathol. 2013, 114, 337-340. [CrossRef] [PubMed]

41. Chrostek, E.; Gerth, M. Is Anopheles gambiae a Natural Host of Wolbachia? mBio 2019, 10, e00784-19. [CrossRef]

42. Ross, P.A.; Callahan, A.G.; Yang, Q.; Jasper, M.; Arif, M.A.K.; Afizah, A.N.; Nazni, W.A.; Hoffmann, A.A. An Elusive Endosymbiont: Does Wolbachia Occur Naturally in Aedes aegypti? Ecol. Evol. 2020, 10, 1581-1591. [CrossRef] [PubMed]

43. Kulkarni, A.; Yu, W.; Jiang, J.; Sanchez, C.; Karna, A.K.; Martinez, K.J.; Hanley, K.A.; Buenemann, M.; Hansen, I.A.; Xue, R. Wolbachia pipientis Occurs in Aedes aegypti Populations in New Mexico and Florida, USA. Ecol. Evol. 2019, 9, 6148-6156. [CrossRef] [PubMed]

44. Osei-Poku, J.; Han, C.; Mbogo, C.M.; Jiggins, F.M. Identification of Wolbachia Strains in Mosquito Disease Vectors. PLoS ONE 2012, 7, e49922. [CrossRef] [PubMed]

45. Wiwatanaratanabutr, I.; Zhang, C. Wolbachia Infections in Mosquitoes and Their Predators Inhabiting Rice Field Communities in Thailand and China. Acta Trop. 2016, 159, 153-160. [CrossRef] [PubMed]

46. Carvajal, T.M.; Hashimoto, K.; Harnandika, R.K.; Amalin, D.M.; Watanabe, K. Detection of Wolbachia in Field-Collected Aedes aegypti Mosquitoes in Metropolitan Manila, Philippines. Parasites Vectors 2019, 12, 361. [CrossRef] [PubMed] 